\title{
Review of the NA48 CP violation measurements with neutral and charged kaons
}

\author{
Patrizia Cenci ${ }^{1}$ \\ INFN Perugia \\ Via A. Pascoli, 06123 Perugia, Italy \\ E-mail: patrizia.cenciepg.infn.it
}

\begin{abstract}
The main results on the $\mathrm{CP}$ violation measurements with neutral and charged kaons from the NA48 experiment at the CERN SPS are reviewed in this paper. The direct CP violating parameter $\operatorname{Re}\left(\varepsilon^{\prime} / \varepsilon\right)$ has been measured by NA48 from the decay rates of neutral kaons into pion pairs. The final result, based on the data collected in 1997, 1998, 1999 and 2001, clearly established the existence of direct $\mathrm{CP}$ violation. NA48 also measured the $\mathrm{CP}$ violation parameter $\left|\eta_{+-}\right|$, defined as the amplitude ratio of CP violating to CP conserving neutral kaon decays into two charged pions: $\left|\eta_{+-}\right|=\mathrm{A}\left(\mathrm{K}_{\mathrm{L}} \rightarrow \pi^{+} \pi^{-}\right) / \mathrm{A}\left(\mathrm{K}_{\mathrm{S}} \rightarrow \pi^{+} \pi^{-}\right)$. The CP violating decay $\mathrm{K}_{\mathrm{S}} \rightarrow \pi^{0} \pi^{0} \pi^{0}$ has been studied by the NA48/1 experiment, using the data taken in 2000 and 2002 with a $\mathrm{K}_{\mathrm{S}}$ beam at high intensity. Only direct $\mathrm{CP}$ violation occurs in charged kaon decays, since mixing is not allowed. NA48/2 measured the Dalitz plot asymmetry of $\mathrm{K}^{ \pm} \rightarrow 3 \pi^{ \pm}$decays from the data collected in 2003 and 2004, setting a new limit on the asymmetry in the decay matrix slope parameters. Based on the same data sample, the result on the search for direct $\mathrm{CP}$ violation in the decay rate asymmetry in $\mathrm{K}^{ \pm} \rightarrow \pi^{0} \pi^{ \pm} \gamma$ processes is also presented.
\end{abstract}

European Physical Society Europhysics Conference on High Energy Physics EPS-HEP 2009,

Krakow, Poland

July 16-22, 2009

\footnotetext{
${ }^{1}$ Speaker, on behalf of the NA48, NA48/1 and NA48/2 Collaborations.
} 


\section{Introduction}

CP violation was discovered in $\mathrm{K}_{\mathrm{L}}^{0} \rightarrow \pi \pi$ decays in 1964 [1]. The same mode provided the evidence for direct CP violation [2-4]. All three types of CP violation are observed in neutral kaons. The phenomenon of neutral kaon mixing accounts for indirect $\mathrm{CP}$ violation measured with the parameter $\operatorname{Re}(\varepsilon)$, where $\varepsilon$ represents the asymmetric mixing of the $\mathrm{CP}$ eigenstates into the mass eigenstates. Direct $\mathrm{CP}$ violation is due to an asymmetry in the amplitude of $\mathrm{K}^{0}$ decays into two pions with different isospin values. This effect is quantified with the parameter $\operatorname{Re}\left(\varepsilon^{\prime}\right)$. A non-zero value of $\varepsilon$ ' arises naturally in the Standard Model (SM) from the complex phase of the CKM matrix. The violation of CP symmetry takes also place in the interference between decays with and without mixing, represented in terms of the parameters $\operatorname{Im}(\varepsilon)$ and $\operatorname{Im}\left(\varepsilon^{\prime}\right)$. Only direct $\mathrm{CP}$ violation effects occur in the charged kaon sector, since mixing is not allowed.

Over the past 10 years, the NA48 experiment at the CERN SPS carried out an extensive physics program devoted to the study of $\mathrm{CP}$ violation. The main results on $\mathrm{CP}$ violation measurements with neutral and charged kaons from NA48, NA48/1 and NA48/2 are presented.

\section{CP Violation in Neutral Kaons}

NA48 was a fixed target experiment at the CERN SPS aiming at the measurement of direct CP violation in the neutral kaon sector. The NA48 detector and its performances are described in [5]. Simultaneous $\mathrm{K}_{\mathrm{L}}$ and $\mathrm{K}_{\mathrm{S}}$ beams, produced by the same primary $450 \mathrm{GeV} / \mathrm{c}$ proton beam, have been exploited. Given the different decay lengths of $\mathrm{K}_{\mathrm{L}}$ and $\mathrm{K}_{\mathrm{S}}$ mesons, two production targets were used, located $126 \mathrm{~m}$ ("far-target") and $6 \mathrm{~m}$ ("near-target") upstream of the decay region. A bent silicon crystal was deflecting by channelling the protons non interacting in the far-target thus defining an attenuated proton beam impinging on the $\mathrm{K}_{\mathrm{S}}$ target. In order to distinguish $\mathrm{K}_{\mathrm{S}}$ and $\mathrm{K}_{\mathrm{L}}$ decays, the protons directed to the $\mathrm{K}_{\mathrm{S}}$ target were tagged by an array of thin scintillator counters. Fig. 1 shows the schemes of the NA48 beam and detector.

Direct CP violation was established in 1999 with the first results of the precision experiments E832 (KTeV) [3] at FNAL and NA48 [4]. The observable was the double ratio:

$$
R=\frac{\Gamma\left(K_{L} \rightarrow \pi^{0} \pi^{0}\right) \cdot \Gamma\left(K_{S} \rightarrow \pi^{+} \pi^{-}\right)}{\Gamma\left(K_{S} \rightarrow \pi^{0} \pi^{0}\right) \cdot \Gamma\left(K_{L} \rightarrow \pi^{+} \pi^{-}\right)}=\left|\frac{\eta_{00}}{\eta_{+-}}\right|^{2} \cong 1-6 \operatorname{Re}\left(\varepsilon^{\prime} / \varepsilon\right)
$$

In order to cancel systematic effects at first order in $R$, the measurement was accomplished by counting events under the following conditions: the $\mathrm{K}_{\mathrm{L}}$ and $\mathrm{K}_{\mathrm{S}}$ beams were produced concurrently and the four $\pi \pi$ decay modes, coincident in space, were measured simultaneously. The two experiments exploit similar detector concepts, with high performance calorimetry for the $\pi^{0} \pi^{0}$ measurement - a liquid Krypton ionization chamber for NA48 and pure CsI crystals for $\mathrm{KTeV}$ - and high quality magnetic spectrometers for the $\pi^{+} \pi^{-}$detection. The final result from NA48 [6], based on the data taken in the years 1997 to 2001 , is: $\operatorname{Re}\left(\varepsilon^{\prime} / \varepsilon\right)=(14.7 \pm 2.2) \times 10^{-4}$. 

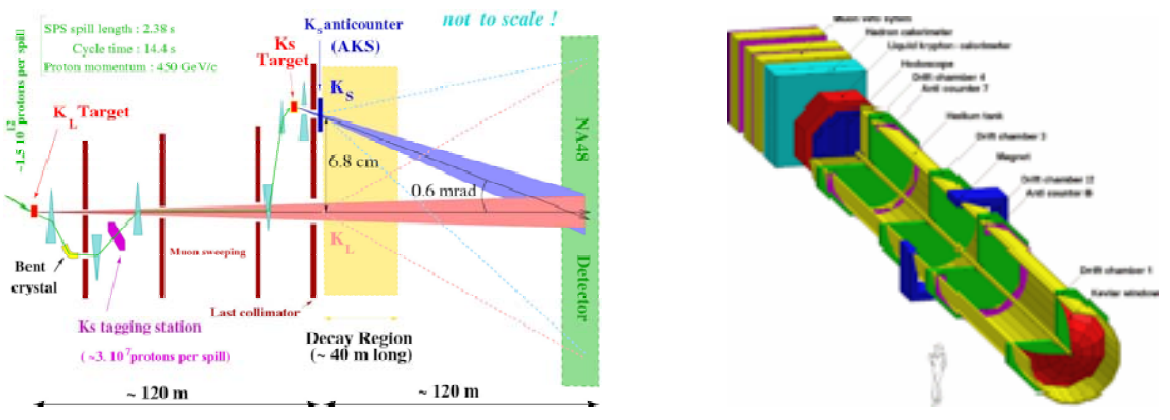

Figure 1: the layout of the NA48 neutral beams (left); the NA48 detector (right).

The amplitude ratio of $\mathrm{CP}$ violating to $\mathrm{CP}$ conserving decays of the neutral kaons into two charged pions is related to the CP violation parameters: $\mid \eta_{+-}=\mathrm{A}\left(\mathrm{K}_{\mathrm{L}} \rightarrow \pi^{+} \pi^{-}\right) / \mathrm{A}\left(\mathrm{K}_{\mathrm{S}} \rightarrow \pi^{+} \pi^{-}\right) \approx \varepsilon^{+} \varepsilon^{\prime}$.

In order to compute the branching ratio of the decay $\mathrm{K}_{\mathrm{L}} \rightarrow \pi^{+} \pi^{-}$, needed to calculate $\left|\eta_{+-}\right|$, the decay rates ratio $\mathrm{R}=\Gamma\left(\mathrm{K}_{\mathrm{L}} \rightarrow \pi^{+} \pi^{-}\right) / \Gamma\left(\mathrm{K}_{\mathrm{L}} \rightarrow \pi \mathrm{ev}\right)$ has been measured in NA48. The final result is $\left|\eta_{+-}\right|=(2.223 \pm 0.012) \times 10^{-3}$ [7]. This value is in agreement with the measurements done by $\mathrm{KTeV}$ [8] and by KLOE [9], while contradicts the 2004 PDG value [10].

The NA48/1 experiment was aiming at the measurement of Ks rare decays with an intense $\mathrm{K}_{\mathrm{S}}$ beam and a near-target modified with respect to the NA48 one [5]. It took data in 2000 and 2002. NA48/1 measured the branching fraction of the CP violating process $K_{S} \rightarrow 3 \pi^{0}$ and the amplitude ratio $\left|\eta_{000}\right|=\mathrm{A}\left(\mathrm{K}_{\mathrm{S}} \rightarrow 3 \pi^{0}\right) / \mathrm{A}\left(\mathrm{K}_{\mathrm{L}} \rightarrow 3 \pi^{0}\right)$ [11]. An indirect method was exploited, based on the measurement of $\mathrm{K}_{\mathrm{S}}-\mathrm{K}_{\mathrm{L}}$ interference effects close to the $\mathrm{K}_{\mathrm{S}}$ production target, achieving an accuracy comparable to the more precise direct measurement done by KLOE [12].

\section{CP Violation in Charged Kaons}

Direct CP violation is expected to induce different amplitudes for $\mathrm{K}^{+}$and $\mathrm{K}^{-}$decays into the same three-pion final states. A non-zero value of the asymmetry of the decay matrix slope parameter $g$, i.e. $A_{g}=\left[g\left(K^{+}\right)-g\left(K^{-}\right)\right] /\left[g\left(K^{+}\right)+g\left(K^{-}\right)\right]$, would indicate direct CP violation effects. In those modes, the slope asymmetry $A_{g}$ is expected to be strongly enhanced with respect to the asymmetry of integrated decay rates. The SM predictions for $A_{g}$ are of the order of $10^{-5}[13]$ or even smaller, while calculations involving processes beyond the SM [14,15] do not exclude enhancements up to few $10^{-4}$. NA48/2 accomplished an extensive study of direct CP violation effects in $\mathrm{K}^{ \pm}$decays into three pions using a modified NA48 experimental setup and a new design of simultaneous, narrow band, non separated $\mathrm{K}^{+} / \mathrm{K}^{-}$beams [16]. The experiment aimed to measure the slope asymmetry $A_{g}$ in $\mathrm{K}^{ \pm} \rightarrow 3 \pi^{ \pm}$and $\mathrm{K}^{ \pm} \rightarrow \pi^{ \pm} \pi^{0} \pi^{0}$ events with a sensitivity of $\mathrm{O}\left(10^{-4}\right)$, one order of magnitude better than the best available measurements.

The simultaneous data taking with $\mathrm{K}^{+} / \mathrm{K}^{-}$beams of similar momentum spectra, associated to the regular reversal of the polarity of the spectrometer magnetic field and of the magnets along the beam line, allow the cancellation of most of the systematic effects due to asymmetries in the setup. Possible local effects on the acceptances due to the electric charge are equalized. Several data samples were collected in all possible combinations of polarities of both the beam line and the spectrometer magnets. This procedure allows to accomplish further cancellation of systematic biases due to differences in the beam line geometry, in the rates and in the detector. 
The method is independent on the $\mathrm{K}^{+} / \mathrm{K}^{-}$flux ratio and on the size of the samples collected under the different conditions. The result is only sensitive to asymmetries in the experimental conditions with time variation scales smaller than those of the magnetic field inversions and it is free of systematic biases. In addition, no Monte Carlo corrections to the acceptance are required.

Based on $3.1 \times 10^{9} \mathrm{~K}^{ \pm} \rightarrow 3 \pi^{ \pm}$decays and $9.1 \times 10^{7} \mathrm{~K}^{ \pm} \rightarrow \pi^{ \pm} \pi^{0} \pi^{0}$ decays, collected in 2003-04, the final results of the measurements of the charged $A_{g}{ }^{c}$ and neutral $A_{g}{ }^{n}$ asymmetries are [17]:

$\mathrm{A}_{\mathrm{g}}^{\mathrm{c}}=\left(-1.5 \pm 1.5_{\text {stat. }} \pm 0.9_{\text {trig. }} \pm 1.3_{\text {syst. }}\right) \times 10^{-4}=(-1.5 \pm 2.2) \times 10^{-4}$

$A^{n}{ }_{g}=\left(1.8 \pm 1.7_{\text {stat. }} \pm 1.7_{\text {syst }}\right) \times 10^{-4}=(1.8 \pm 1.8) \times 10^{-4}$

The accuracy achieved by NA48/2 is one order of magnitude better than the previous measurements. The results are compatible with the SM and do not show evidence of direct CP violation, giving constrains to SM extensions which predict enhancements of the asymmetries.

The interference component of the $\mathrm{K}^{ \pm} \rightarrow \pi^{ \pm} \pi^{0} \gamma$ processes has been measured by NA48/2 for the first time [18]. No evidence of direct $\mathrm{CP}$ violation has been found in the measurement of the $\mathrm{K}^{ \pm} \rightarrow \pi^{ \pm} \pi^{0} \gamma$ decay rate asymmetry $\mathrm{A}_{\mathrm{N}}=\left[\Gamma\left(K^{+}\right)-\Gamma\left(K^{-}\right)\right] /\left[\Gamma\left(K^{+}\right)+\Gamma\left(K^{-}\right)\right]$. Based on a sample of $1.08 \times 10^{6}$ events, a new limit has been set by NA48/2: $\mathrm{A}_{\mathrm{N}}<1.5 \times 10^{-3}$ (90\% C.L.).

\section{References}

[1] J. H. Christenson et al., Phys. Rev. Lett. 13, 138 (1964).

[2] H. Burkhardt et al., Phys. Lett. B 206, 169 (1988).

[3] A. Alavi-Harati et al., Phys. Rev. Lett. 83, 22 (1999).

[4] V. Fanti et al., Phys. Lett. B 465, 335 (1999).

[5] V. Fanti et al., Nucl. Inst. Methods A 574, 433 (2007).

[6] J.R. Batley et al., Phys. Lett. B 544, 97 (2002).

[7] A.Lai et al., Phys. Lett. B 645, 26 (2007).

[8] T. Alexopoulos et al., Phys. Rev. D 70, 092006 (2004).

[9] F. Ambrosino et al., Phys .Lett. B 638, 140 (2004).

[10] S. Eidelman et al., Particle Data Group, Phys .Lett. B 592, 1 (2004).

[11] A.Lai et al., Phys. Lett. B 610, 165 (2005).

[12] F. Ambrosino et al., Phys. Lett. B 619, 61 (2005).

[13] E. Gámiz et al, JHEP 10, 42 (2003).

[14] E.P. Shabalin, ITEP preprint 8-98 (1998).

[15] G. D’Ambrosio Phys. Lett. B 480, 164 (2000).

[16] J.R. Batley et al., Phys. Lett. B 649, 349 (2007).

[17] J.R. Batley et al., Eur. Phys. J. C 52, 875 (2007).

[18] E. Marinova, these proceedings. 